\title{
The Psychological Nursing of Patients with Infectious Diseases
}

\author{
Liu Libing ${ }^{1, a}$, Liu Fang'e ${ }^{2, b}$, Hua Qianzhen ${ }^{3, c}$, Wu Jin ${ }^{4, d}$ \\ ${ }^{1}$ Medical School, Xi'an Peihua University, 710125, China \\ ${ }^{2}$ Medical School, Xi'an Peihua University, 710125, China \\ ${ }^{3}$ Medical School, Xi'an Peihua University, 710125, China \\ ${ }^{4}$ Medical School, Xi'an Peihua University, 710125, China \\ a 357435305@qq.com, ${ }^{b}$ liufange@126.com, ${ }^{c}$ 3034658577@qq.com, ${ }^{\mathrm{e}}$ 1359011404@qq.com
}

Keywords: psychological nursing; Patients ;infectious diseases;

\begin{abstract}
Patients with infectious diseases have their unique psychological characteristics, which differ from those of other kinds of patients. Psychological nursing, as a new approach, can fully exert subjective initiative and accelerate the rehabilitation process. Psychological nursing, as an effective treatment of patients with infectious diseases, plays an increasingly more important role in hospitals and modern life.
\end{abstract}

\section{Introduction}

Infectious diseases, caused by a variety of pathogens, can be transmitted among humans and animals. They often came with the developing human civilization, and have a lasting profound effect on human beings. Sometimes, the influence of infectious diseases is even worse than the influence of warfare, revolution, or insurrection. Infectious diseases, in varying degrees: can decrease a population, reduce production efficiency, and even affect the evolution of human civilization. They can be transmitted through blood, saliva, breast milk, semen and feces through direct and indirect contact. Due to the fear of becoming infected, people almost always attempt to excessively isolate themselves from or discriminate against patients with infectious diseases, and this can lead to patients developing psychological problems. Such psychological problems will often aggravate their illness. Therefore we conclude that scientific psychological nursing often has an important positive influence on the recovery of patients with infectious diseases [1].

\section{Psychological Characteristics of Patients with Infectious Diseases}

In the long human history, many catastrophic events have happened, but there is no catastrophe that has caused such disastrous consequences as infectious disease has done. Today, even with the enormous developments of science and technology, infectious diseases are still a threat to human health. Because of there special properties, infectious diseases have there special psychological characteristics.

Fear and Insecurity. These feelings often emerge in the early stage and treatment of the disease. Many young patients worry that for example their disease cannot be cured; or that they will become infected with an even more serious infectious disease; or that their relatives and friends will ignore and reject them. Some even hide their illness for fear of the treatment, or when diagnosed request medical staff to keep secret the nature of their illness for fear of isolation from family and friends.[2].

Pessimism and Despair. This phenomenon is more common in patients with long duration severe illness and poor economic circumstances. Because of the long-term pain, their poor economic position makes their circumstances particularly difficult to bear, resulting in negative speculations and a heavy burden of emotional instability producing pessimism and despair [2]. Patients with HIV/AIDS, for example, directly their illness is diagnosed face a severe psychological blow and from there on fall into a very negative psychological state. [3]. 
Anxiety. Anxiety psychology is more common in patients with chronic infectious diseases, mostly amongst the youth and women. Because of the long periods of treatment and the risks, or their experience, of disease relapse patients often feel anxiety distress. So during the period of treatment a patient is emotionally unstable, easily excited and depressed. Some patients doubt whether there is progress with their recovery from infection and whether their drugs have been matched properly or effectively. Some young patients worry about their personal future particularly their career and capability of social interaction. Female patients continually worry about their family and particularly their children.. Many patients often ask the medical staff about the results of laboratory tests. Most patients want to be discharged as soon as possible [2].

Depression and Autism. The incidence rate of depression in patients with infectious diseases is significantly higher than in the general population. To some degree, patients with a severe depression may contemplate suicide. These patients are characterized by low mood phases, long periods of silence and imaginative activity unrelated to reality. According to the report of Xie Haili, STD patients' social avoidance and distress measurement scored significantly higher than that of healthy people [4].

Lonely. Because infectious diseases are contagious, severe cases of infection often need a long isolation treatment, which requires them to be separated from their relatives. Some patients cannot fully understand the requirement for the isolation regime, and consequently feel the medical staff and their relatives are alienating themselves from them with motives which the patients in their imaginations find very distressing. This kind of lonely moody thinking can seriously affect the process and even the effectiveness of the patients' treatment [5].

Irritability. Due to the long duration of chronic infectious disease, patients may more easily relapse. Moreover, expensive medical bills make patients feel guilty towards their families; some may even feel that they are a very heavy burden. Such patients may show the negative emotion of irritability [6].

Excessive worry. Fearing becoming infected with other kind of infectious disease, some patients dare not touch objects within the ward or even walk around. Thus they have a serious lack of security

Indifference. Some patients give up on the possibility of recovery because they lack some basic understanding of the disease they have. They might not follow their doctor's advice, and say the prescribed sterile barrier system this can create more difficulties for their effective treatment [7].

\section{Psychological Nursing Measures for Patients with Infectious Diseases}

In the process of the treatment of infectious diseases, it is necessary for the medical staff to administer effective psychological nursing. Taking positive and effective nursing measures to eliminate patients' negative emotions benefits the rehabilitation of patients and the treatment of there infectious diseases. In order to avoid harm to others and themselves medical staff can take suitable treatment when necessary [6].

Create a Good Environment for Medical Treatment. In order to relieve the patient's worries, medical staff should from the firstly try to make a clean, tidy, quiet and comfortable environment and leave a good first impression. Then, the medical staff can take this and every opportunity to establish a good communication with patients, such as introducing there treatment, diet, visiting times and prohibitions and so on. It is very helpful to eliminate the worries of patients and their families at the start and indeed whenever possible.[8].

Effective Communication with Patients. The medical staff should introduce the specific conditions and treatment of infectious disease to the patients and always give patients positive stimuli. During the treatment, the medical staff should keep an eye on the psychological fluctuations of the patients, particularly helping them to eliminate or at least reduce psychological anxiety and fear. Moreover, they can even teach the patients how to control their bad mood swings and keep a positive attitude to their treatment. At the same time, staff can give genuine testimonies of where past patients have completed successful treatment and been discharged and perhaps on what 
conditions, this can greatly enhance patients' confidence to conquer themselves and their disease. Last but not least, in a ward, staff should very carefully arrange the situation of the different types of 'infectious disease patients' to eliminate the risk of cross infection; and also make sure that patients, their families and other visitors are aware of the care that has been taken and any procedures they themselves should take.

Humanistic Care in Nursing. Nursing staff should have good professional ethics accomplishment and professional skills. When communicating with patients nursing staff should use the gentle language to make the patients feel at ease, and that they are respected, as this helps them feel self-confident. In the process of implementing nursing operations, nursing staff ought to pay attention to the protection of a patient's privacy, select an appropriate time to enlighten patients and help them cooperate with treatment actively. Meanwhile, nursing staff should mind their body language such as their gestures, facial expressions, and attitudes. For example, with the care of depressed patients, a concerned communication and caring expression or handshake can cheer them up [8].

Focus on Personality. There are no two identical leaves in the world! So even identical human twins won't have the same character! Medical staff should know about each of their patients' individual personality, and be briefed and observe their psychological condition; always being familiar with their medical records or other daily communications, so keeping to an appropriate plan for each patient [1].

Contribution from the Patient's Family. Throughout the process of treatment, the relationship between patients and their family members is very important as this can greatly affect the efficacy of treatment. The medical staff should let their families know that their care, frequent visitations and heart-to-heart talks will encourage patients and give them great support. At the same time, in order to eliminate a visitor's fear and any risk of getting infected, the medical staff should teach some basic knowledge about infectious diseases, such as the importance of disinfection and isolation and some tips about preventing infection.

Health Education. After admission, nursing staff should immediately explain some basic knowledges of and tips for a patient's daily life. These are some measures: (1) provide a health education handbooks; (2) broadcast related videos on the prevention and treatments of infectious diseases; (3) introduce as appropriate relevant infection knowledge, such as the pathogen, susceptible population, routes of transmission, clinical manifestations, etc. Make patients master their knowledge of their own infectious disease through explanation and cooperation with their own treatment. (4) On discharge give guidance such as paying attention to the personal hygiene, avoiding public activities, quitting unhealthy behavior, etc.

Effective Ongoing Contact Discharge Guidance. The medical staff should provide each patient with a detailed contact card including appointment times, their doctor's name and phone number, etc; this is necessary but also can ease the anxiety states of patients and their families.

Regular Visitor Experience Record. Medical staff should establish a book of family visits and appointments; so recording and trying to understand the patient's psychological status through the debriefing of at least some of those visiting. This book should perhaps be private to the staff, and others be unaware of it.

Medical Care. Psychological care should be combined with medical care to get a better effect. For example, in order to avoid the cross infection, the medical staff will take some measures such as frequently wiping the floor with disinfectant, and additionally disinfecting the ward and corridor with an ultraviolet lamp perhaps weekly; and so by doing this very obviously and carefully improve all the patients' confidence.

\section{Differences between Psychological Intervention and Non-intervention}

A study found that patients with psychological intervention are significantly better served with the relieving of anxiety and worries than those without intervention as groups. $(\mathrm{P}<0.05)$ Meanwhile, the nursing satisfaction is about 7\% higher than with the non-intervention group [9]. Another study implied by Yu Shumin has come to the same conclusion. To sum up, in the treatment 
of patients with infectious disease, the medical staff should take measures to eliminate negative emotions to achieve the best nursing effectiveness. [8]. After nursing intervention, patients' cognition on infectious disease have been improved obviously $(\mathrm{P}<0.01)$ and social avoidance and distress significantly decreased $(\mathrm{P}<0.01)$, which shows that the implementation of comprehensive nursing intervention for infectious diseases can improve the patients' awareness of their disease, alleviate their psychological pressures, reduce the degree of social avoidance and distress and improve the quality of an infectious disease patients life.

\section{Conclusion}

From what has been set out above, we can see that patients with infectious diseases always have negative emotions such as anxiety, irritability and worries, which are unfavorable to their treatment. As a result of their disease, most patients with infectious diseases are extremely sensitive to information from outside and the behavior and language of the medical staff in the daily life of the hospital.

Therefore, it is necessary to take scientific psychological intervention to the patients with infectious diseases. Through a series of psychological interventions, the patients can fully feel the concern from qualified people which helps establishing their confidence and so helps cure their disease. Besides, psychological intervention can effectively relieve the negative emotions of patients, and help promote the conditions for recovery. In addition, during the implementation of psychological intervention, the nursing staff's professional skills and their personal qualities can be improved, which will bring to patients more kindness and improved quality services, which will help avoid medical disputes between the staff, the patients and their relatives.

\section{References}

[1] Bai Lijuan, Wu Wen, Psychological Intervention of Anxiety and Depression in Patients with Infectious Diseases. Chin J Mod Drug Apply, 20159 (9) 240.

[2] Du Guixin, Psychological Nursing on Patients with Infectious Diseases, Practical medical journal, 1997 4(7) 544-545.

[3] Huang Lianxin, Nong Xiaozhen, Huang Xiaozhen, Luo Suzhen, Li Wenxin, Comprehensive Nursing Intervention On the Social Avoidance and Distress of Patients with HIV/AIDS, Chinese Youjiang Medical Journal 2015, Vol.43 No.1,116-117.

[4] Xie Haili, Li Huimin, Ma Guowei, Reaserch on AIDS and Venereal Disease in China, 2013 19(2) 140-141.

[5] Du Shuli, Li Xiufeng, Zhang Bingyan, Psychological Characteristics of Patients With Infectious Diseases, Chin J Mod Drug Apply, 2010 4(13) 260.

[6] Ai Hua, Psychological Nursing Intervention for Patients with Infectious Diseases [J]. Chinese Community Doctors, 2015 31(57) 157.

[7] Wang Hui, Wang Yafeng, Zhang Shibo, Yang Lei, The Psychological Characteristics of Patients with Chronic Infectious Diseases and Nursing Experience [J], China Practical Medicine, 2014 9(16) 195-196.

[8] Yu Shumin, Yu Yang, Zhang Xiaoying, Lu Xuemin, Zhang Hongyun, Effective Nursing Experience in Patients with Digestive Infectious Diseases, China Practical Medicine, 2015 10(15) 221.

[9] Sun Guijuan, Wang Lihua, Observations of the Psychological Nursing Intervention on the Negative Emotion of Patients with Infectious Diseases, Chin J Mod Drug Apply, 2015 9(5) 216. 